\title{
Corela
}

Cognition, représentation, langage

HS-7 | 2010

Espace, Préposition, Cognition

\section{Une explication cognitive des effets attribués à la c-commande dans les contraintes sur la coréférence}

Denis Bouchard

\section{OpenEdition}

Journals

Édition électronique

URL : http://journals.openedition.org/corela/965

DOI : 10.4000/corela.965

ISSN : 1638-573X

Éditeur

Cercle linguistique du Centre et de l'Ouest - CerLICO

Référence électronique

Denis Bouchard, «Une explication cognitive des effets attribués à la c-commande dans les contraintes sur la coréférence », Corela [En ligne], HS-7 | 2010, mis en ligne le 31 mai 2010, consulté le 02 mai 2019. URL : http://journals.openedition.org/corela/965 ; DOI : 10.4000/corela.965

Ce document a été généré automatiquement le 2 mai 2019.

\section{(c) (i) (2)(2)}

Corela - cognition, représentation, langage est mis à disposition selon les termes de la licence Creative Commons Attribution - Pas d'Utilisation Commerciale - Partage dans les Mêmes Conditions 4.0 International. 


\title{
Une explication cognitive des effets attribués à la c-commande dans les contraintes sur la coréférence
}

\author{
Denis Bouchard
}

\section{NOTE DE L'AUTEUR}

Je remercie deux relecteurs de CORELA pour leurs commentaires fort utiles.

1 La linguistique cognitive porte une attention particulière à l'incidence de la pensée sur la langue et accorde un rôle essentiel à la conceptualisation du monde dans l'étude de la langue. Dans ce cadre de pensée, il est intéressant de déterminer quel modèle de conceptualisation est pertinent pour une étude linguistique, et d'autre part, jusqu'où peut s'étendre l'explication de propriétés langagières par des propriétés cognitives.

2 Les travaux de Claude Vandeloise ont abordé la première question et démontré que la sémantique de certains éléments linguistiques, qui semblait de prime abord relever de caractéristiques d'une physique naïve de la perception de l'espace, dépend en fait de fonctions cognitives beaucoup plus complexes (voir Vandeloise 1986, 1987, 1988, 1992, $1995,1999,2003)$. Dans cet article, j'aborde la seconde question. Je discute de contraintes sur la coréférence, comme celles qui sont suggérées par des phrases comme en (1) :

(1)a. Rumsfeld affirme qu'il ne sait pas d'où proviennent ces armes.

(1)b. Il affirme que Rumsfeld ne sait pas d'où proviennent ces armes. où Rumsfeld et $i l$ peuvent être coréférentiels en (1a) mais pas en (1b).

3 Ces données ont fait l'objet de très nombreuses études en grammaire générative. Pour rendre compte des faits, on propose une condition sur les expressions référentielles comme celle en (2) (voir l'exposition classique du problème et la Condition $C$ de la théorie du liage dans Chomsky 1981).

(2) Condition sur les expressions référentielles: 
Une expression référentielle doit être libre.

Libre $_{\text {déf }}$ : Une expression est libre si elle n'est pas c-commandée par un élément portant le même indice de référence.

C-commande ${ }_{\text {déf }}$ : A c-commande B si le premier nœud branchant qui domine A domine aussi B en structure syntaxique.

4 Le pronom il c-commande Rumsfeld en (1b), d'où l'impossibilité d'une interprétation coréférentielle d'après la condition en (2). Cette condition sur les expressions référentielles est présentée comme un principe spécifique au langage qui est encodé dans la Grammaire Universelle (GU), c'est-à-dire ce que les générativistes présument être un module inné, présent à la naissance chez tout être humain. C'est précisément à cette propriété d'innéité de la condition que l'on fait appel pour rendre compte du fait que les enfants ne font pas d'erreurs dans l'acquisition de relations de coréférence comme en (1) : voir Crain et Pietroski (2006) (parmi bien d'autres) pour une exposition de cet argument fondé sur la pauvreté de stimulus. Mais comme le phénomène porte sur la référence, qui est une notion relevant de la situation pragmatique d'énonciation, on peut s'interroger sur la justesse d'en rendre compte par une condition inscrite dans la GU, d'autant plus que la clé de cette condition est la c-commande. Comme cette notion fait partie de la composante syntaxique, présumée autonome en grammaire générative, sa présence semble tout à fait accidentelle dans une condition d'une tout autre nature, portant sur les possibilités de référence : on ne voit pas la motivation d'une telle relation structurale.

Une raison additionnelle de douter d'une analyse syntaxique du phénomène vient de données comme en (3) et (4), où Rumsfeld et il ne peuvent pas être coréférentiels.

(3)Bush a louangé Rumsfeld, et il l'a fait aussi.

(4)Locuteur $\mathrm{A}: \mathrm{Je}$ sais où $i \mathrm{l}$ cache des armes de destruction massive.

Locuteur B : Moi aussi. Dans le garage de Rumsfeld.

6 On peut à la rigueur imaginer une reconstruction de structure pour rendre compte de l'impossibilité de coréférence en (3), suivant des analyses de phrases semblables en anglais. Ainsi, la reconstruction en Forme Logique de la phrase anglaise (5) serait comme en (6), où too est remplacé par le syntagme verbal de la phrase principale (voir Crain et Pietroski 2006) :

(5) Bush praised Rumsfeld, and he did too.

(6) Bush praised Rumsfeld, and he did praise Rumsfeld.

7 Suivant ce modèle de reconstruction, la Forme Logique de la phrase (3) aurait un contenu semblable à celui de la phrase en (3'), tandis que la Forme Logique de l'énoncé du locuteur B en (4) serait comme en (4').

(3') Bush a louangé Rumsfeld, et il a louangé Rumsfeld aussi.

(4') Je sais qu'il cache des armes de destruction massive dans le garage de Rumsfeld.

Dans ces deux cas, le pronom il c-commande l'expression référentielle Rumsfeld, ce qui contrevient à la condition en (2). Toutefois, cette stratégie de reconstruction pour sauver l'analyse semble bien peu probable quand l'effet se produit entre des énoncés de locuteurs différents comme en (4), puisque les opérations de reconstruction de structure en Forme Logique appartiennent à la syntaxe de phrase. ${ }^{1}$

L'approche strictement computationnelle amène à présenter les faits de telle manière qu'il devient impossible de les expliquer, et qu'il faut donc lister la condition dans la GU. On constate qu'il y a une corrélation entre la relation syntaxique de c-commande et certains cas de dépendances de référence, mais on ne peut expliquer pourquoi cette 
notion est en jeu plutôt que n'importe quelle autre. Ainsi, le système serait tout aussi cohérent si la définition de Libre en (2) était remplacée par celle en (2') :

(2') Libre $2_{\text {déf }}$ : Une expression est libre si elle est c-commandée par un élément portant le même indice de référence.

10 Une liste de conditions comme la GU permet de décrire les faits, mais pas de les expliquer: ce n'est qu'une assertion d'existence, un inventaire généralisé de faits inexpliqués. Chomsky (2007: 19) reconnaît d'ailleurs que la GU est le résidu contenant les éléments inexpliqués de l'état initial du locuteur. Je vais montrer qu'au moins dans le cas de phénomènes de dépendance référentielle comme ceux discutés ici, on peut expliquer les propriétés langagières par des propriétés cognitives et par la sémantique élémentaire de la phrase, ce qui permet de sortir de l'impasse d'une liste de conditions basées sur des notions contingentes comme la c-commande.

11 La relation syntaxique en jeu est, en fait, le signifiant d'un signe combinatoire. En regardant le côté signifié plutôt que signifiant de cette relation, sa présence dans une contrainte sur ce type de dépendance découle naturellement de propriétés sémantiques de la prédication et de propriétés pragmatiques de la référence. Il devient alors possible de proposer une explication basée sur le processus cognitif en jeu dans ce phénomène de coréférence. Déjà en 1979, Thomas Wasow suggérait une piste de solution. Il a remarqué que la Condition $C$ «semble être une condition qui pourrait découler d'une contrainte cognitive plus générale - quelque chose qui aurait pour effet que l'interprétation d'un élément ne peut dépendre d'un élément moins informatif» (Wasow 1979: 36) [ma traduction]. Il propose la contrainte en (7) à cet effet.

(7) Condition de Nouveauté : Un élément anaphoriquement dépendant ne peut avoir une référence plus déterminée que son antécédent.

12 Le degré de détermination de la référence d'un élément dépend de la quantité d'information qu'il fournit quant à l'identification de référents possibles. La détermination de la référence dépend donc de la situation et ne relève pas seulement de la sémantique mais aussi de la pragmatique. La Condition de Nouveauté est une condition cognitive et on s'attend à ce qu'elle s'applique indépendamment de toute construction linguistique particulière, et même au-delà du langage : si on identifie un élément en se servant d'information provenant d'un autre élément, cet autre élément doit fournir plus d'information que l'élément dépendant. ${ }^{2}$ On peut aussi, comme le propose Levinson (2000), relier l'emploi linguistique de la condition à la maxime pragmatique de Grice "N'en dis pas plus que nécessaire ", qui fait en sorte qu'un élément référentiellement dépendant sera sémantiquement plus général.

13 La condition de Wasow rend compte de données comme les suivantes, où docteur produit un SN qui a un plus haut degré de détermination que le SN ayant homme comme tête :

(8)a. Un docteuri est entré dans la pièce. L'hommei ne dit rien au début.

(8)b *Un hommei est entré dans la pièce. Le docteuri ne dit rien au début.

14 Une contrainte comme la Condition de Nouveauté a un double avantage sur la Condition C. D'abord, elle a une plus grande couverture empirique: elle rend compte aussi directement d'exemples comportant des relations anaphoriques internes à une phrase que d'exemples comportant des relations anaphoriques externes à la phrase, tandis que la Condition C ne s'applique à des cas comme (3)-(4) et (8) qu'avec des élaborations complexes et peu vraisemblables de structures syntaxiques furtives. De plus, la Condition de Nouveauté ouvre la voie à une explication des faits fondée sur des propriétés cognitives indépendamment motivées portant sur le contenu informatif relatif. ${ }^{3}$ À 
l'opposé, la Condition C est une contrainte spécifique au langage qui doit être listée dans la GU, sans explication fondée. Cette condition ne fait que décrire quels éléments sont impliqués (des pronoms et des expressions référentielles) et quelle configuration bloque certaines interprétations (c-commande). Il n'y a aucune raison fondée indiquant pourquoi cette relation structurale se retrouve dans une condition sur l'interprétation référentielle, plutôt que n'importe quelle autre relation structurale, ni pourquoi la condition s'applique dans cette direction (pourquoi c'est le pronom qui ne peut ccommander l'expression référentielle plutôt que l'inverse), ni pourquoi même il y a une telle condition. On doit stipuler tout ces éléments parce qu' ils ne découlent de rien. ${ }^{4} \mathrm{En}$ revanche, la Condition de Nouveauté permet de fonder des stratégies essentielles de l'interprétation sémantique et pragmatique qui ne pourraient être inversées.

Les pronoms sont des éléments dont l'interprétation référentielle dépend typiquement d'une autre expression référentielle. La Condition de Nouveauté saisit l'intuition qu'une expression référentielle ne peut dépendre d'un pronom pour son interprétation puisque l'expression référentielle a une référence plus déterminée que le pronom. Il faut donc comprendre de quelle façon l'expression référentielle dépend du pronom pour son interprétation dans des exemples comme ceux en (3)-(4) et (8). La Condition C indique qu'il y a une relation de proéminence qui est impliquée. Mais comme la coréférence est une propriété pragmatique, qui dépend aussi de propriétés sémantiques des éléments, la condition de proéminence devrait être de nature interprétative plutôt que syntaxique. De plus, il devrait y avoir une raison claire expliquant pourquoi la proéminence en question a un effet sur l'interprétation des dépendances référentielles.

Dans les exemples en (3)-(4) et (8), le pronom est un sujet. En plus de sa relation référentielle, un pronom sujet est, par définition, impliqué dans une relation sémantique manifeste : le sujet est l'argument de la prédication du syntagme verbal. Or le SV de ces exemples contient des expressions référentielles explicites ou implicites. Si l'on regarde la façon dont se fait l'interprétation de ce SV, on a une indication de ce qui crée le problème d'interprétation de la coréférence. Comme le prédicat complexe du SV est appliqué au sujet, ce prédicat dépend de l'interprétation référentielle du sujet, en ce sens que la validité de cette prédication dépend de l'identité du sujet, de ses référents possibles. Le problème soulevé par les exemples en (3)-(4) et (8), c'est que l'interprétation de la prédication dépend de l'identité du pronom sujet, et en même temps, l'identité du pronom sujet dépend de l'identité d'un syntagme nominal qui fait partie du SV prédicatif. L'interprétation du pronom sujet et l'interprétation du SV prédicatif sont donc croisées, dépendantes l'une de l'autre, de sorte que les interprétations sont insolubles, incohérentes, s'il y a coréférence entre le pronom sujet il et l'expression Rumsfeld. Cette explication suggère une façon plus perspicace d'exprimer la condition de liage.

(9) Théorème sur la dépendance référentielle :

Un élément référentiellement dépendant $\mathrm{E}$ (comme un pronom) ne peut dépendre d'une expression référentielle qui est dans le constituant avec lequel il entretient une relation de prédication.

Il important de noter que (9) n'est pas une condition de la grammaire mais un théorème qui découle d'autres aspects de la grammaire indépendamment motivés, qui produisent ici une interprétation incohérente. De plus, ce théorème explique pourquoi la condition sur le liage est négative : c'est parce que l'interaction entre les relations référentielles et la relation prédicative produit une interprétation incohérente dans les cas en question. Enfin, notons qu'avec ce théorème, la notion de c-commande ne joue aucun rôle dans 
l'analyse. Il se trouve que la relation de prédication entre le SV et le sujet correspond à une relation configurationnelle de c-commande dans certains modèles syntaxiques. Mais une configuration syntaxique ne crée pas de problème d'interprétation. Le problème vient d'une incohérence d'interprétation entre le sujet et le prédicat dans certains contextes : c'est donc dans la relation prédicative elle-même que se trouve l'explication plutôt que dans la façon de l'exprimer en syntaxe.

L'analyse prédit que moins un pronom fournit d'information concernant l'identité du référent du sujet, plus ce pronom pourra être référentiellement dépendant d'un SN qui fait partie du SV prédicatif, puisqu'il y a alors un croisement de dépendance atténué entre l'interprétation du sujet et l'interprétation du SV prédicatif. Ainsi, un pronom qui est enchâssé dans le sujet plutôt que d'être lui-même le sujet fournit beaucoup moins d'information quant à l'identité du référent du sujet, de sorte que des phrases comme en (10) n'entrent pas en conflit avec le théorème en (9), ni avec la Condition de Nouveauté.

(10)a. Qu'on l'élimine de la course a surpris Paul.

(10)b. Sa mère a fait la bise à Jean. ${ }^{5}$

Voyons maintenant un autre exemple où on a fait appel à la relation structurale de ccommande pour expliquer des contraintes sur l'interprétation coréférentielle. Il s'agit d'un cas particulier de liage de pronoms réfléchis discuté par Jackendoff (1992) et Culicover et Jackendoff (2005), comme dans l'exemple en (11) dans une situation impliquant Ringo et une statue de Ringo: cette phrase ne peut être interprétée que comme en (a) et non comme en (b).

(11) Ringo fell on himself.

(11)a. Ringo est tombé sur la statue de Ringo.

(11)b. $\neq$ La statue de Ringo est tombée sur Ringo.

À noter, l'équivalent français de (11) n'est guère acceptable et un locuteur produirait plus vraisemblablement (11'b) plutôt que (11'a).

(11')a. ? ? Ringo est tombé sur lui-même.

(11)b. Ringo est tombé sur une/la statue qui le représente / à son effigie.

Les phrases en (11a) et (11'a) sont des transgressions éventuellement humoristiques et les analyser exige un contexte, comme me l'a fait remarquer un relecteur de CORELA. Mais malgré le fait qu'une condition discursive soit en jeu ici, comme dans les autres exemples discutés, j'utilise des exemples fabriqués (par moi-même ou d'autres auteurs) sans fournir de contexte d'utilisation parce que ce n'est pas nécessaire pour rendre compte du type de dépendance référentielle visée par une condition comme celle en (2), ou le théorème en (9). Cela évite de porter flanc à une critique de formalistes qui pourraient prétendre que l'analyse sémantico-cognitive est trop éloignée de l'analyse basée sur la c-commande, qu'elle ne traite pas des mêmes propriétés des données, et qu'elle est peut-être un complément à la c-commande mais pas une solution de rechange. Ces critiques pourraient arguer qu'en situation d'énonciation, un énoncé peut même en venir à dire le contraire du sens calculé à partir de ses éléments lexicaux et de leur combinaison par des règles morphosyntaxiques, comme dans des cas d'ironie, etc.: les conditions pragmatiques nous éloigneraient donc trop des effets visés par la condition (2). Je veux montrer qu'une analyse alternative et supérieure est possible même en restant près d'une grammaire de la phrase, en ajoutant seulement des conditions très générales d'une grammaire de l'énoncé requises pour l'analyse de la référence, et sans entrer dans les détails d'un contexte spécifique. 
21 Revenons à l'exemple (11). Le liage du pronom réfléchi dépend de l'interprétation référentielle des éléments, et il n'y a rien dans la syntaxe de surface qui permet de faire la distinction entre les interprétations en (11a) et (11b). Culicover et Jackendoff (2005: 228) suggèrent que les exemples de ce genre impliquent une sorte de coercition : un morceau additionnel de sens peut être optionnellement inséré dans la représentation sémantique pour aider à ce qu'elle fasse sens.

On pourrait proposer une analyse syntaxique de ce fait basée sur une structure sousjacente reconstruite sur le modèle des phrases en (11a-b), en supposant que la statue de est effacé sous certaines conditions. Supposons une condition de liage stipulant qu'un pronom réfléchi doit être c-commandé localement par son antécédent. Au niveau où s'appliquent les conditions de liage, Ringo serait alors profondément enchâssé en (11b) et ne pourrait c-commander et lier le pronom réfléchi, d'où l'agrammaticalité avec l'interprétation «statue». Toutefois, Culicover et Jackendoff rejette cette analyse : «le fait que la règle "statue " soit hautement contrainte par des facteurs sémantiques idiosyncratiques suggère qu'elle ne peut pas être une composante de la syntaxe autonome » (p. 367; ma traduction). Par exemple, les mots anglais picture et sketch peuvent être utilisés pour référer à un objet physique ou à une description plus abstraite ; cependant, la règle «statue » ne s'applique que dans le deuxième cas. De plus, l'analyse syntaxique rencontre des problèmes d'accord du sujet avec le verbe et d'assignation de cas du sujet. On peut ajouter qu'il y a aussi un problème de constituance : la séquence effacée la statue de n'est pas un constituant syntaxique. Culicover et Jackendoff proposent plutôt de rendre compte de la restriction sur l'interprétation de (11) en posant une règle de coercition qui agit sur les interfaces (leur règle (40) p. 371) :

(12) La structure syntaxique SN peut correspondre à la structure sémantique/ conceptuelle REPRÉSENTATION PHYSIQUE $(\mathrm{X})$, où $\mathrm{X}$ est l'interprétation ordinaire de SN.

Cette approche requiert que la théorie du liage soit dépendante à la fois de configurations sémantiques et de configurations syntaxiques. À l'appui de leur hypothèse, Culicover et Jackendoff présentent quelques constructions additionnelles où le liage est contraint par la sémantique. Ils proposent que des coercitions semblables affectent le liage pour des verbes qui expriment des transferts d'information comme mention, ou qui expriment des représentations mentales comme show:

(13) *I mentioned Ringo to himself.

[CAUSE (I, [GO ([INF $\left.\left(\operatorname{RINGO}^{\alpha}\right)\right]$, TO $\left.\left.\left.(\alpha)\right]\right)\right]$

(14) *I showed John to himself.

[CAUSE (I, [GO ([REP (JOHN $\left.\left.{ }^{\alpha}\right)\right]$, TO (MIND ( $\left.\left.\left.\left.\left.\left.\alpha\right)\right)\right)\right]\right)\right]$

24 Ces structures conceptuelles empêchent le liage, selon Culicover et Jackendoff, à cause d'un équivalent à la c-commande qui s'applique en structure conceptuelle. Le liage d'un pronom réfléchi peut aussi dépendre de notions discursives comme la connexion logophorique en (15a), où l'attitude ou la volonté de l'antécédent est exprimée dans la deuxième partie de la phrase; ceci contraste avec l'exemple (15b), où il n'y a pas de connexion logophorique. ${ }^{6}$

(15)a. Another picture of himself in the paper and Susan thinks John will definitely be offended.

(15)b. Another picture of him(*self) in the paper and Susan divorces John.

Culicover et Jackendoff (p.482) disent qu'ils ne voient pas bien comment expliquer la différence d'acceptabilité de himself entre ces deux phrases. En fait, il y a des conditions cognitives sur la pragmatique de la référence qui ont été beaucoup étudiées et qui 
expliquent de façon unifiée tous les phénomènes ci-dessus où il y a apparemment une forme de coercition, et ce, sans ajouter quelque matériel ad hoc que ce soit aux représentations. Comme le note Coppieters $(1982,1990)$ et les références qu'il cite, une entité peut être présentée d'un point de vue externe, neutre, ou d'un point de vue interne. Si on la présente de façon externe, l'entité est traitée comme un Concept, tandis que présentée de façon interne, l'entité est traitée comme un Substantif, c'est-à-dire une entité qui peut être un participant dans un événement. De plus, il y a une sous-classe de Substantifs qui peuvent être présentés comme des Sujets Intentionnels, des entités conscientes de l'événement. Ceci est résumé en (16), avec un exemple de chacun des trois cas en (17), où Jean est un Concept en (17a), un Substantif en (17b), et un Sujet Intentionnel en (17c).

(16)Concept : 1 . point de vue externe, neutre par rapport à l'entité

2. jugement objectif du locuteur, narrateur omniscient

Substantif : 1 . point de vue interne

2. entité comme participant

Sujet-I : 1 . point de vue interne, sentiments/émotions de l'entité

2. entité est consciente de l'événement (Sujet de Conscience)

(17)a. Jean est un type qui est très confiant. (Concept)

(17)b. Jean a attrapé un rhume. (Substantif)

(17)c. Jean n'est pas sûr de bien connaître Marie. (Sujet-I)

La distinction entre Concept (point de vue externe) et Substantif (point de vue interne) est motivée indépendamment parce qu'elle détermine le choix entre les pronoms il (ou elle) et ce/ça. Coppieters $(1982,1990)$ montre qu'ils correspondent à des façons différentes de référer à un $\mathrm{SN}$ instigateur (sujet) : ce est employé pour exprimer un point de vue externe, tandis que il est employé pour référer à l'entité d'un point de vue interne ; voir aussi Cadiot (1988), entre autres.

Dans Bouchard (1995), je montre que ces notions ont un rôle important dans le liage des pronoms réfléchis. En particulier, lorsque les pronoms réfléchis sont «à longue distance", ils ne se comportent pas comme de véritables anaphores mais comme des pronoms logophoriques (Zribi-Hertz 1989), et ils doivent alors référer au Sujet de Conscience minimal, comme l'exprime la condition en (18) (Bouchard 1995 : 299).

(18) Liage à distance des pronoms réfléchis ${ }^{7}$

Un pronom réfléchi à distance peut être lié par un Sujet de Conscience si ce pronom

est dans le Contenu de Conscience de ce Sujet de Conscience.

Un Sujet de Conscience est un individu auquel le locuteur prête une conscience, tandis qu'un Contenu de Conscience est ce que le locuteur présente comme faisant partie de la conscience d'un individu. Selon (18), un pronom réfléchi à distance est donc lié par un Sujet de Conscience si le pronom est dans une partie de la phrase dont le sens est présenté par le locuteur comme faisant partie du Contenu de Conscience de cet individu. Cette condition rend compte de différences dans le liage «à rebours" comme dans les exemples anglais suivants :

(19)a. That book about herself struck Mary as embarrassing.

(19)b. *That book about herself struck Mary on the head.

En (19a), Mary peut lier herself parce que herself est dans le Contenu de Conscience du Sujet Intentionnel Mary, parce que dans l'emploi «psychologique» de struck, Mary est présentée comme étant consciente que le contenu du livre la concerne, porte sur elle. En (19b), Mary ne peut pas être l'antécédent du pronom réfléchi parce que that book about herself est un Substantif, un objet concret, et son contenu n'est pas présenté comme 
faisant partie du Contenu de Conscience de Mary. Les effets de liage qui dépendent de ce genre de notions pragmatiques sont décrits en détail dans Bouchard (1995), chapitre 4, particulièrement en ce qui concerne les expressions dites "psychologiques» (qui expriment des sentiments, des émotions). Ainsi, la condition (18) s'étend aux exemples en (15) : la phrase contenant himself est présentée comme faisant partie du Contenu de Conscience de John en (15a), mais pas en (15b). La condition (18) rend également compte des faits en (11) et en (13)-(14). Ainsi, en (11), Ringo-statue ne peut être l'antécédent du pronom réfléchi puisqu'il n'est pas présenté comme un Sujet de Conscience. On peut généraliser l'analyse encore plus: si l'on fait l'hypothèse naturelle que référer à un élément en tant que Concept procure une référence moins déterminée que référer à un élément en tant que Substantif, alors l'interprétation en (11b) est rejetée par la Condition de Nouveauté de Wasow en (7), puisque ce que Culicover et Jackendoff représente comme [REP (RINGO)] correspond à un Concept. Ceci renforce le pouvoir explicatif de l'analyse puisqu'elle repose sur une condition cognitive qui vaut en général et pas seulement pour le langage. ${ }^{8}$

30 Les notions dans les conditions (7) et (18), de même que celles qui déterminent le choix entre les pronoms il et ce, relèvent de manières de référer, donc du discours, de la pragmatique, plus que de la sémantique. En conséquence, même si l'on suppose qu'il y a un niveau de représentation de sémantique conceptuelle avec des structures comme en (13) et (14) comme le proposent Culicover et Jackendoff, il faut néanmoins déterminer si certains « morceaux de sens » comme REPRÉSENTATION PHYSIQUE (X) ou INFORMATION (X) sont nécessaires. D'après ce que nous avons vu ci-dessus, ça ne semble pas être le cas. Non seulement ces structures cachées ne sont pas nécessaires, elles ne semblent pas non plus suffisantes. Ainsi, d'après Culicover et Jackendoff, ces morceaux de structure bloquent le liage à cause d'un équivalent à la c-commande en structure sémantique/ conceptuelle. Toutefois, il ne semble pas y avoir d'équivalent à la c-commande qui soit à l'œuvre dans des exemples comme (15) ou (19): l'antécédent ne c-commande pas le pronom réfléchi (à moins d'introduire des structures ad hoc dans la représentation sémantique par des artifices qu'eux-mêmes rejettent fortement en syntaxe). D'autres exemples confirment l'inadéquation d'une approche structurale, comme ceux en (20) (tirés de Pesetsky 1990), où les antécédents John et Mary sont enchâssés dans des positions qui ne c-commandent pas le pronom réfléchi.

(20)a These rumors about himselfi caught Johni's attention.

(20)b The rumors about herselfi made Maryi's hair stand up.

31 Les analyses structurales, que ce soit en syntaxe ou en sémantique, ne sont pas du tout naturelles pour ces cas, alors qu'une analyse basée sur des conditions de discours fournit une explication générale et naturelle des faits que Culicover et Jackendoff attribuent à des coercitions. Ces faits dépendent de conditions pragmatiques/inférentielles plutôt que de syntaxe ou de sémantique. Ceci vaut en général pour les analyses basées sur des coercitions, y compris pour l'exemple phare de coercition en (21) (Beard 1991; Pustejovsky 1991, 1995 ; Jackendoff 1997).

(21) Marie a commencé un livre. [ = Marie a commencé À LIRE un livre]

Dans Bouchard (2002), je montre que dans ce cas aussi ce sont des notions non linguistiques qui sont à l'œuvre. Le type de composition active qu'on postule pour des exemples comme (21) n'opère pas sur des sens en fait, mais sur des éléments que nous inférons à partir de notre connaissance des référents impliqués. ${ }^{9}$ On le voit dans le fait que la même composition active se produit dans des exemples comme en (22). 
(22) J'ai/Il a/Elle a commencé Dostojevsky.

Comme les pronoms et les noms propres n'ont pas de propriétés sémantiques comme les qualia de Pustejovsky $(1991,1995)$, la composition active ne peut pas se faire en sémantique ici. Cette composition relève plutôt de la pragmatique. Ainsi, afin d'interpréter correctement l'apport à la signification de commencé en (22), il faut savoir à quoi Dostojevsky réfère et connaître certaines propriétés encyclopédiques de ce référent, et de plus connaître des propriétés du référent de je, il, elle (si cette personne est un lecteur, un relieur, un éditeur, un traducteur, un peintre, un metter en scène, etc.).

En conclusion, on voit à travers ces divers exemples d'utilisation de la notion syntaxique de c-commande dans l'analyse de phénomènes ayant trait à la coréférence que la voie tracée par des linguistes cognitivistes comme Claude Vandeloise conduit à des stratégies d'analyses qui permettent d'éliminer d'apparentes aberrations comme une relation structurale qui affecterait un phénomène discursif comme la référence. Du même coup, on peut entrevoir des explications véritables fondées sur des propriétés cognitives indépendamment motivées là où on n'avait qu'une liste de contraintes contingentes qu'on devait supposées inscrites dans le cerveau humain, faute d'être capable de relier ces éléments de la Grammaire Universelle à des principes explicatifs indépendants. Il ressort que les très fréquentes disparités entre syntaxe et sémantique pour lesquelles des linguistes inventent des reconstructions et des coercitions sont en fait des conséquences du système d'analyse qu'ils ont adopté, des effets secondaires d'une présentation erronée des faits. Il en va de même d'une notion comme la c-commande, dont l'usage abusif découle d'une méthode d'analyse où l'on recherche des éléments qui sont absolument spécifiques au langage et exagérément formels.

Cet article n'est qu'une esquisse d'analyse qui ne fait qu'effleurer l'ensemble des propriétés des dépendances référentielles, et il y a une quantité d'autres phénomènes à prendre en compte. Mon but est beaucoup plus modeste que de faire une analyse exhaustive des faits, mais il est néanmoins fort important. Le type d'analyse proposée montre que des réponses s'appuyant sur des notions cognitives sont plus éclairantes que celles qui sont fortement orientées vers les aspects computationnels, et qu'on peut échapper à l'impasse descriptiviste de l'approche formelle. Non seulement cette analyse est plus explicative parce que fondée sur des notions indépendamment motivées, mais, en outre, elle se généralise à plus de phénomènes plus diversifiés, et canalise tout autant l'acquisition du langage. ${ }^{10}$

\section{BIBLIOGRAPHIE}

Banfield, Ann. 1982. Unspeakable Sentences. London : Routhledge et Kegan Paul.

Beard, Robert. 1991. Decompositional Composition : The Semantics of Scope Ambiguities and 'Bracketing Paradoxes'. Natural Language and Linguistic Theory 9 : 195-229.

Bouchard, Denis. 1995. The Semantics of Syntax. Chicago : The University of Chicago Press. 
Bouchard, Denis. 2002. Adjectives, Number and Interfaces : Why Languages Vary. Oxford : Elsevier Science.

Cadiot, Pierre. 1988. De quoi ça parle A propos de la référence de ça, pronom-sujet. Le Français Moderne 56 : 174-192.

Cantrall, William. 1974. Viewpoint, Reflexives, and the Nature of Noun Phrases. The Hague : Mouton.

Chomsky, Noam. 2007. Of Minds and Language. Biolinguistics $1: 9-27$.

Chomsky, Noam. 1981. Lectures on Government and Binding. Foris Publications, Dordrecht

Coppieters, René. 1982. Descriptions and attitudes : The problem of reference to individuals. Studies in Language $6: 1-22$.

Coppieters, René. 1990. Individuals, thematic roles, and Semantic Interpretation. Pomona College.

Crain, Stephen, and Pietroski, Paul. 2006. Is Generative Grammar deceptively simple or simply deceptive. Lingua $116: 64-68$.

Culicover, Peter, and Jackendoff, Ray. 2005. Simpler Syntax. Oxford : Oxford University Press.

Epstein, Samuel David, and Seely, T. Daniel. 2002. Derivation and Explanation in the Minimalist Program. Oxford : Blackwell.

Hagège, Claude. 1974. Les pronoms logophoriques. Bulletin de la Société Linguistique de Paris 69 : 287-310.

Higgins, Francis. 1979. The Pseudo-Cleft Construction in English. New York : Garland Press.

Jackendoff, Ray. 1992. Madame Tussaud meets the binding theory. Natural Language and Linguistic Theory $10: 1-31$.

Jackendoff, Ray. 1997. The architecture of the language faculty. Cambridge, MA : MIT Press.

Keenan, Edward. 1976. Towards a Universal Definition of 'Subject'. In Syntax and Semantics : Subject and Topic, ed. Charles Li, 303-333. New York : Academic Press.

Kuno, Susumo. 1972. Pronominalization, Reflexivization, and Direct Discourse. Linguistic Inquiry $3: 161-195$.

Kuno, Susumo. 1975. Three Perspectives in the Functional Approach to Syntax. In Papers from the Parasession on Functionalism, ed. Robin Grossman, 276-336. Chicago : Chicago Linguistic Society.

Kuno, Susumo. 1983. Reflexivization in English. Communication and Cognition 16:65-80.

Kuno, Susumo. 1987. Functional Syntax : Anaphora, Discourse and Empathy. Chicago : University of Chicago Press.

Kuroda, S.-Y. 1965. Generative Grammatical Studies in the Japanese Language, Department of Linguistics, Massachusetts Institute of Technology.

Kuroda, S.-Y. 1973. Where Epistemology, Style, and Grammar Meet : A Case Study from Japanese. In A Festschrift for Morris Halle, eds. Steven Anderson and Paul Kiparsky, 377-391. New York : Holt, Rinehart et Winston.

Lakoff, George. 1970. A Note on Ambiguity and Vagueness. Linguistic Inquiry 1 : 357-359.

Levinson, Stephen. 2000. Presumptive Meaning: The theory of Generalized Conversational Implicature. Cambridge, MA : MIT Press. 
Pesetsky, David. 1990. Experiencer Predicates and Universal Alignment Principles. Ms. Cambridge, MA.

Pollard, Carl, and Sag, Ivan. 1992. Anaphors in English and the Scope of Binding Theory. Linguistic Inquiry $23: 261-303$.

Pustejovsky, James. 1991. The Generative Lexicon. Computational Linguistics 17.

Pustejovsky, James. 1995. The Generative Lexicon. Cambridge, MA : MIT Press.

Ruwet, Nicolas. 1990. EN et Y : Deux clitiques pronominaux antilogophoriques. Langages 97 : 51-81.

Sells, Peter. 1987. Aspects of Logophoricity. Linguistic Inquiry 18 : 445-479.

Uriagereka, Juan. 2002. Derivations. London : Routledge.

Vandeloise, Claude. 1986. L'espace en français : sémantique des prépositions spatiales. Paris : Seuil (Travaux Linguistiques).

Vandeloise, Claude. 1987. La préposition à et le principe d'anticipation. Langue Française, 76 : 77-111.

Vandeloise, Claude. 1988. Les usages statiques de la préposition à. Cahiers de Lexicologie, 53 : 119-148.

Vandeloise, Claude. 1992. Les analyses de la préposition dans : faits linguistiques et effets méthodologiques. Lexique $11: 15-40$

Vandeloise, Claude. 1995. De la matière à l'espace : la préposition dans. Cahiers de Grammaire 20 : 123-145.

Vandeloise, Claude. 1999. Quand dans quitte l'espace pour le temps. Revue de Sémantique et Pragmatique 6 : 145-162

Vandeloise, Claude. 2003. Langues et cognition. Editions Hermès, Paris.

Wasow, Thomas. 1979. Anaphora in Generative Grammar. Gent : E. Story-Scientia.

Zribi-Hertz, Anne. 1989. Anaphor Binding and Narrative Point of View : English Reflexive

Pronouns in sentence and discourse. Language 65 : 695-727.

\section{NOTES}

1. De plus, Culicover et Jackendoff (2005, chapitre 7) présentent plusieurs cas semblables où la structure syntaxique qu'il faut reconstruire ne correspond pas à une phrase acceptable en anglais, comme dans les deux exemples suivants : (i)A : Ozzie loves Harriet., (i)B : But not himself/* him [*But not Ozzie loves himself.], (ii) Ozzie doesn't love Harriet, let alone himself/*him. [*...let alone Ozzie loves himself.]

2. La Condition de Nouveauté a des traits communs avec la notion d'autonomie de référence proposée par Keenan (1976 : 98) : un SN est référentiellement autonome lorsqu'il est utilisé pour référer à un objet ou un concept dont le référent est identifiable pour l'allocutaire au moment de l'énoncé. La référence d'un tel SN ne peut dépendre de la référence d'un autre SN qui suit dans le discours.

3. Uriagereka (2002: 56) reconnaît aussi qu'il vaut mieux traiter de la théorie du liage en sémantique et en pragmatique plutôt qu'en syntaxe. Il propose entre autres d'analyser l'effet de croisement faible ("weak crossover ») en (i) comme une violation de la condition de nouveauté/ 
familiarité et que le his familier ne peut antécéder le nouveau a man en (i), pas plus qu'il ne peut le faire en (ii): (i) His ${ }_{i}$ friend killed a man ${ }_{i}$; (ii) His friend knocked on the door. A man ${ }_{i}$ came in. Étonnamment, Uriagereka ne cite pas le travail de Wasow (1979).

4. Epstein et al. (2002) disent qu'il y a une raison naturelle à l'ubiquité de la c-commande en grammaire: cette relation structurale indique que les objets syntaxiques peuvent entrer en relation avec des éléments de la portion de structure avec laquelle ils sont combinés. Mais cela ne répond à aucune des trois questions que nous venons d'évoquer concernant la condition sur les relations de coréférence.

5. Je considère que « l'adjectif possessif » sa est essentiellement un pronom au génitif.

6. En bref, un pronom logophorique doit avoir comme antécédent un élément porteur d'un rôle discursif, comme par exemple le sujet de conscience, c'est-à-dire la personne dont on présente la pensée, le discours ou le sentiment (Hagège 1974, Kuno 1987, Zribi-Hertz 1989).

7. L'inspiration pour cette condition m'est aussi venue de Kuroda $(1965,1973)$, Kuno $(1972,1975$, 1983, 1987), Cantrall (1974), Banfield (1982), Sells (1987), Ruwet (1990), Pollard et Sag (1992).

8. Cette analyse s'étend à un vaste domaine empirique. Ainsi, Bouchard (1995: 300) mentionne que la condition (18) peut aussi rendre compte d'autre cas de connectivité entre un pronom réfléchi et un antécédent distant comme ceux discutés par Higgins (1979) : (i)a. Pictures of himself, John knows Bill likes.; (i)b. Which pictures of herself did Mary buy? De plus, Ruwet (1990) indique que ces notions discursives procurent aussi une explication pour la distribution des pronoms en et $y$, qu'il analyse comme des pronoms antilogophoriques qui sont soumis à la condition discursive suivante: (ii) Si en ou $y$ se trouvent dans une proposition exprimant un contenu de conscience $\mathrm{CCi}$, en et $y$ ne peuvent être coréférentiels du N" qui représente le sujet de conscience SCi de ce CCi (Ruwet 1990 : 8). Ainsi, Émile ne peut lier en en (iii.a) parce que en fait partie de son Contenu

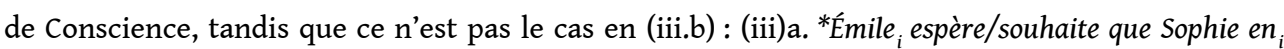
tombera amoureuse.; (iii)b. Émile ${ }_{i}$ mérite que Sophie en ${ }_{i}$ tombe amoureuse. Bouchard (1995 : 379-382) montre comment une légère modification de la condition (ii) permet de rendre compte des possibilités d'extraction de ne en italien et de en en français hors des objets directs «Expérienceurs» dans les constructions psychologiques. Ainsi, l'extraction est relativement acceptable en (iv), mais impossible en (v) (où $t$ indique la position d'extraction de ne/en dans une analyse transformationnelle) : (iv)a. Gianni ne teme [il presidente $t$ ]; (iv)b. Jean en craint [le président $t$ ]; (v)a. *Questa situazione ne preoccupa [il presidente $t$ ]; (v)b. *Cette situation en préoccupe [le président $t$ ].

9. Voir à ce sujet A Note on Ambiguity and Vagueness de Lakoff (1970) et le long débat qu'il a engendré.

10. Crain et Pietroski (2006) répète l'assertion fréquente que la Condition $C$ est un principe unificateur qui s'étend à diverses constructions. On dit que ceci renforce l'hypothèse selon laquelle ce principe fait partie de notre fondement inné. Toutefois, la généralité de la condition se fait au prix de reconstructions fort lourdes dans plusieurs cas. De plus, un système plus simple, plus général n'a pas à être inné, et vice versa. Chomsky a souvent dit que la simplicité est une propriété du système langagier qui est intéressante précisément parce qu'elle est inattendue, étant donné que les systèmes biologiques sont souvent embrouillés (évidemment, tout dépend du niveau où l'on se place pour observer : à un certain niveau, un cœur peut sembler n'être qu'un fouillis de molécules, mais paraît bien structuré comme organe pour pomper du sang). Dans la perspective biolinguistique adoptée par Chomsky depuis plusieurs décennies, il n'y a aucune raison de s'attendre à ce qu'un principe de la Grammaire Universelle se généralise à plusieurs phénomènes : chaque phénomène pourrait tout aussi bien dépendre d'un principe indépendant listé en GU. L'acquisition du langage serait tout autant contrainte qu'avec un seul principe unificateur, puisque les principes de la GU, qu'ils soient unifiants ou spécifiants, n'ont pas à être appris selon cette hypothèse. 


\section{RÉSUMÉS}

On retrouve la notion de c-commande dans plusieurs contraintes linguistiques sur l'interprétation d'éléments qui sont dépendants pour leur interprétation référentielle. À bien y penser, il est assez étonnant d'avoir une relation syntaxique comme contrainte sur ce type d'interprétation : cela semble tout à fait accidentel, on n'en voit pas la motivation. Cette relation syntaxique correspond en fait le signifiant d'un signe combinatoire, et en regardant le côté signifié plutôt que signifiant de cette relation, sa présence dans une contrainte sur la dépendance référentielle découle naturellement de propriétés sémantico-conceptuelles de la référence et la prédication.

The notion of c-command occurs in several constraints on the interpretation of elements which are dependent on others for the interpretation of their reference. However, it is rather surprising that a syntactic relation should constrain referential interpretation : it seems totally accidental, there is no clear motivation for it. That syntactic relation actually corresponds to the signifiant of a combinatorial sign, and if we look at the signifié side of that relation instead of its signifiant, its presence in a constraint on referential dependency follows naturally from semantic/conceptual properties of reference and predication.

INDEX

Mots-clés : c-commande, référence, dépendance, signe, prédication

Keywords : c-command, dependency, sign

\section{AUTEUR}

\section{DENIS BOUCHARD}

Université du Québec à Montréal 